\title{
Evaluations of the Chuanqi Ophthalmic Microemulsion In Situ Gel on Dry Age-Related Macular Degeneration Treatment
}

\author{
Maobo Du, ${ }^{1}$ Shuo Shen $\left(\mathbb{D},{ }^{1}\right.$ Lina Liang, ${ }^{2}$ Kai Xu, ${ }^{2}$ Aiping He $\mathbb{D},{ }^{1}$ Yao Yao $\mathbb{D},{ }^{1}$ \\ and Shuzhi Liu $\mathbb{D}^{1}$ \\ ${ }^{1}$ Institute of Chinese Materia Medica, China Academy of Chinese Medical Sciences, Beijing, China \\ ${ }^{2}$ Eye Hospital, China Academy of Chinese Medical Sciences, Beijing, China \\ Correspondence should be addressed to Shuzhi Liu; liushuzhi2004@sina.com
}

Received 3 May 2020; Revised 28 June 2020; Accepted 21 July 2020; Published 19 August 2020

Academic Editor: Juntra Karbwang

Copyright (c) 2020 Maobo Du et al. This is an open access article distributed under the Creative Commons Attribution License, which permits unrestricted use, distribution, and reproduction in any medium, provided the original work is properly cited.

\begin{abstract}
Age-related macular degeneration (AMD) is the third largest eye disease. However, the eye has a variety of drug delivery barriers, which prevent the drug from reaching the lesions in the posterior segment of the eye, coupled with the pathogenesis of dry-AMD; these lead to the lack of effective treatment drugs for dry-AMD. Therefore, the developments of a suitable therapeutic drug and a novel ophthalmic preparation are of great significance for the treatment of dry-AMD. The purposes of this study were to construct a novel traditional Chinese medicine (Chuanqi Fang) anti-AMD microemulsion in situ gel for treating dry-AMD and investigate its characteristic, efficiency, irritation, and tissue distribution. In this study, the characteristic of the Chuanqi microemulsion in situ gel was measured by dynamic light scattering. The electroretinogram (ERG) indicators and the number of retinal pigment epithelial cells were measured to evaluate the therapeutic effect of the novel ophthalmic nanopreparations. Irritation was evaluated according to Technical Guideline Principles (ZGPT4-1). The analysis of tissue distribution was carried out with LC-MS. The research showed that the particle size of microemulsion was $38.56 \pm 0.21 \mathrm{~nm}$. The Chuanqi microemulsion in situ gel had certain roles in repairing retina damage of the dry-AMD animal model and showed no irritation. The tissue distribution study found that the microemulsion in situ gel could effectively deliver the drug to the posterior eye of the AMD model rat through the route of cornea-vitreous body-retina. In conclusion, this study provided a meaningful research strategy and research basis for the development of new dry-AMD therapeutic drugs.
\end{abstract}

\section{Introduction}

Age-related macular degeneration (AMD) is the third largest eye disease all over the world according to the report Vision 2020 [1] released by the World Health Organization (WHO). According to the presence or absence of choroidal neovascularization (CNV), AMD can be divided into two types [2]: dry-AMD and wet-AMD. The eye has a special anatomical structure, forming a variety of drug delivery barriers, including the tear barrier, conjunctiva, corneal barrier, and blood-eye barrier. These barriers prevent the drug from reaching the lesions in the posterior segment of the eye and limit the efficacy of drugs for treating the ocular posterior segment eye diseases (AMD). Due to the special physiological structure and pathogenesis of the eye, there is no clear treatment method and ideal drug treatment for dry-AMD treatment. Therefore, the selection of a suitable therapeutic drug and the preparation of a novel ophthalmic preparation for the efficient delivery of the posterior eye are of great significance for the treatment of dry-AMD.

Traditional Chinese medicine (TCM) has many advantages for treating AMD [3-5]; the TCM for "nourishing Qi" and "activating blood" can often achieve good curative effects for the treatment of fundus diseases. Among them, Ligusticum chuanxiong and Astragalus membranaceus are the two most commonly used TCMs [6, 7]. Modern ophthalmological pharmacological studies have also found that Ligusticum chuanxiong could treat retinal vein occlusion [8], and Astragalus membranaceus could reduce retinal ischemia-reperfusion injury, and it also had a significant 
protective effect on the retinal ganglion injury model $[9,10]$. According to the clinical experience of TCM, Ligusticum chuanxiong and Astragalus membranaceus can be combined into a compound prescription named Chuanqi Fang. The appropriate dosage form, which is a good treatment for dryAMD, can play the efficacy of Chuanqi Fang.

In the design of the dosage form, prolonging the retention time of the drug in the eye, overcoming a variety of drug delivery barriers, and increasing the concentration of the drug in the posterior segment of the eye are the keys to exert the therapeutic effect of the drug on fundus oculi diseases. At present, the commonly used methods of posterior ophthalmic administration include local administration and intraocular administration. The multiple intraocular injections could increase the risks of vitreous haemorrhage, retinal detachment, and endophthalmitis [11], so the patients' acceptance of intraocular injection is low. It is of great significance to develop a new type of preparation which is comfortable and effective for local treatment of dryAMD. Therefore, in this paper, microemulsion was used to enhance the drug concentration in the posterior eye. Combined with the technical advantages of microemulsion and in situ gel, a series of studies on a new ophthalmic nanopreparation, Chuanqi microemulsion in situ gel, were carried out.

Microemulsion was first named by Schulman [12]. It is composed of water phase, oil phase, surface active agents (SA), and co-surface active agents (Co-SA). Microemulsion has many advantages: (1) enhancing the solubility of different hydrophobic drugs; (2) promoting the biomembrane permeation; (3) improving the bioavailability of drugs; (4) prolonging the release time of water-soluble drugs; and (5) no irritation [13-15]. Therefore, in this paper, microemulsion is very suitable as a carrier for drugs to improve the ability of drugs to cross the barrier and promote the drugs to reach the posterior ocular segment. In situ gel is a special gel which has sol-gel reaction phenomenon, while above the critical point, it transfers from solution to gelatin; however, below the critical point, it transfers from gel to solution. According to the transferring factors, it can be divided into thermosensitive in situ gel [16], pH-sensitive in situ gel [17], and ion-sensitive in situ gel [18]. Among them, the temperature-sensitive in situ gel is very suitable for the development of ophthalmic preparations, which has the advantages of prolonging corneal retention time, accurate dosing, and good reproducibility [19-21]. Based on the advantages of two formulations of microemulsion and in situ gel, the thermosensitive ophthalmic microemulsion in situ gel was constructed in this paper. Compared with the ordinary in situ gel, the microemulsion in situ gel can not only improve the spreading effect of drugs on the ocular surface and the retention time of the cornea but also enable the drug to rapidly penetrate the blood-eye barrier with the help of microemulsion, so as to achieve effective treatment of posterior segment diseases.

In summary, Chuanqi microemulsion in situ gel could meet a series of technical needs for dry-AMD treatment. Therefore, the study established Chuanqi microemulsion in situ gel for the treatment of dry-AMD. Furthermore, nanocarrier characteristics of microemulsion, the efficiency of treating dry-AMD, the irritation, and tissue distribution of the Chuanqi microemulsion in situ gel were evaluated. This study provided a very meaningful research strategy and research basis for the development of new dry-AMD therapeutic drugs.

\section{Materials}

Ligusticum chuanxiong Hort. and Astragalus membranaceus (Fisch.) Bge. var. mongholicus (Bge.) were purchased from Beijing Qiancao Chinese Herbal Medicine Co., Ltd. (Beijing, People's Republic of China). Poloxamer 407, oleic acid (OA), polyoxyethylene hydrogenated castor oil (RH-40), polyethylene glycol 400 (PEG 400), and propylene glycol (PG) were all purchased from BASF (Ludwigshafen, Germany). Gelatin was purchased from Qinghai Gelatin Co., Ltd. (Xining, People's Republic of China). Phosphotungstic acid was purchased from Sigma-Aldrich Co. LLC (Beijing, People's Republic of China). Hematoxylin (HE) was purchased from Sigma-Aldrich Co. LLC (Beijing, People's Republic of China). Ligustrazine, ligustilide, and astragaloside IV were purchased from Wuhan Tianzhi Biotechnology Co., Ltd. (Wuhan, People's Republic of China).

2.1. Animals. Sprague Dawley rats (SD rats) weighing $160 \pm 10 \mathrm{~g}$ were purchased from Beijing Vital River Laboratory Animal Technology Co., Ltd. (Production license no. SCXK (Jing) 2016-0006); the rats were raised in the animal barrier room. The feeding conditions were maintained at $21^{\circ} \mathrm{C}-23^{\circ} \mathrm{C}, 40-70 \%$ relative humidity, and $12 \mathrm{~h}$ dark/light cycle. Experiments were performed after the rats were allowed to acclimate for 7 days.

Male New Zealand rabbits weighing $3 \pm 0.5 \mathrm{~kg}$ were purchased from Beijing Xinglong Experimental Animal Breeding Farm (Production license no. SCXK (Jing) 20160003); the rabbits were raised in the ordinary animal room. The feeding conditions were maintained at $21^{\circ} \mathrm{C}-23^{\circ} \mathrm{C}$. Experiments were performed after the rabbits were allowed to acclimate for 3 days.

All animal experiments in the study were carried out in accordance with the guidelines approved by the Animal Ethics Committee of Eye Hospital, China Academy of Chinese Medical Sciences, Beijing, China.

\section{Methods}

3.1. Preparation of the Chuanqi Extract. The preparation process of the Chuanqi extract was as follows: (1) Ligusticum chuanxiong Hort. and Astragalus membranaceus (Fisch.) Bge. var. mongholicus (Bge.) Hsiao were extracted with $85 \%$ ethanol three times for 1.5 hours per time, and the extraction solvent was recovered by decompression to obtain the Chuanqi ethanol extract; (2) the residue after alcohol extraction was extracted with water three times for 2.0 hours per time. The water extract was concentrated and alcoholprecipitated. The alcohol precipitation supernatant was evaporated to obtain the Chuanqi water extract. 
3.2. Preparation of the Chuanqi Microemulsion In Situ Gel. The composition of the Chuanqi microemulsion in situ gel is shown in Table 1. The preparation process of the Chuanqi microemulsion in situ gel is as follows: (1) blank microemulsion and Chuanqi ethanol extract were stirred with a magnetic stirrer (C-MAG HS4, IKA Works GmbH \& Co, Germany) at $600 \mathrm{rpm}$ for $15 \mathrm{~min}$ to obtain Chuanqi microemulsion; (2) blank in situ gel and Chuanqi water extract were stirred with a mixer (power control-visc, IKA Works $\mathrm{GmbH} \& \mathrm{Co}$, Germany) at $200 \mathrm{rpm}$ for $20 \mathrm{~min}$ to obtain the in situ gel part; and (3) Chuanqi microemulsion part was mixed into the in situ gel part, and the mixture was stirred with a mixer (power control-visc P4, IKA Works $\mathrm{GmbH} \& \mathrm{Co}$, Germany) at $200 \mathrm{rpm}$ for $20 \mathrm{~min}$ to obtain the Chuanqi microemulsion in situ gel [22].

3.3. Preparation of the Chuanqi In Situ Gel. Chuanqi in situ gel was prepared by mixing the Chuanqi ethanol extract, Chuanqi water extract, and blank in situ gel at $200 \mathrm{rpm}$ for $20 \mathrm{~min}$. The composition of the Chuanqi in situ gel is shown in Table 1.

3.4. Characterizations of the Chuanqi Microemulsion In Situ $\mathrm{Gel}$. The $0.2 \mathrm{~g}$ sample (prepared with the method given in “3.2 Preparation of Chuanqi Microemulsion In Situ Gel”) was diluted 500 times with water, and this diluted sample was stirred; the diluted solution was filtered through a filter paper and $0.22 \mu \mathrm{m}$ millipore filter successively. The particle size and zeta potential of Chuanqi microemulsion in the subsequent filtrate were evaluated by dynamic light scattering (Zetasizer Nano-ZS; Malvern Instruments, Malvern, UK) $[23,24]$.

3.5. Morphological Observation of Microemulsion. The $0.2 \mathrm{~g}$ sample (prepared with the method given in "3.2 Preparation of Chuanqi Microemulsion In Situ Gel”) was diluted 500 times with water, and this diluted sample was stirred with a magnetic stirrer and was filtered using the $0.22 \mu \mathrm{m}$ millipore filter. About $0.1 \mathrm{ml}$ of the diluted sample was further diluted 100 times with water. A drop of the final diluted solution was adsorbed on a 400-mesh copper grid, and the excess solution was removed. The sample was air-dried for 10 hours, and then the morphology of microemulsion was observed and photographed by a JEM-2200FS transmission electron microscope $(200 \mathrm{kV})$ (JEOL, Tokyo, Japan).

3.6. Determination of Phase Transition Temperature of the Chuanqi Microemulsion In Situ Gel. The rheological method was used to investigate the microemulsion-in situ gel phase transition temperature. The test parameters of the MCR-102 rheometer (Anton Paar, Austria) were set as follows: initial holding time: $120 \mathrm{~min}$, shear force: $100 \mathrm{~Pa}$, and temperature scanning range: $0^{\circ} \mathrm{C}-40^{\circ} \mathrm{C}$.

3.7. Evaluation of Efficacy Based on the Dry-AMD Animal Model. The establishment of the dry-AMD animal model was the key to evaluate the efficacy. Chemical damage modeling was one of the easiest and most successful methods, especially for sodium iodide modeling; sodium iodate could selectively cause RPE layer damage and then lead to pathological changes in photoreceptor cells and other tissues, which were similar to the pathogenesis of human dry-AMD [25-29]. Therefore, the method of sodium iodide modeling was used to establish the dry-AMD model. On this basis, the ERG indicators (dark ERG, max ERG, bright ERG, and $30 \mathrm{~Hz}$ flicker ERG) and the number of RPE cells were measured to evaluate the therapeutic effect of the novel ophthalmic nanopreparations on the dry-AMD animal model.

3.8. Model Establishment and Grouping. $160 \pm 10 \mathrm{~g}$ male SD rats were used to establish the dry-AMD animal models by a tail vein injection of $40 \mathrm{mg} / \mathrm{kg}$ sodium iodide solution. Before the experiment, all the rats had strict eye examinations to ensure that the rats were free of eyeball and retinal damage. After the modeling, the rats were randomly divided into four groups: Chuanqi microemulsion in situ gel group, model control group, esculin and digitalis glycosides eye drops group, and normal control group (normal rats without modeling). The administration was initiated on the first day after modeling, and the rats were observed at 7 days after the initiation of administration.

The administration was as follows: Chuanqi microemulsion in situ gel was administered into the eye by using the dropper 4 times per day, and the administration time was 9:00, 12:00, 15:00, and 18:00, respectively; model control group: normal saline was administered into the eye by using the dropper 4 times per day, and the administration time was 9:00, 12:00, 15:00, and 18:00, respectively; esculin and digitalis glycosides eye drops group: esculin and digitalis glycosides eye drops were administered into the eye by using the dropper 4 times per day, and the administration time was 9:00, 12:00, 15:00, and 18:00, respectively; normal control group: the animals in this group were fed normally, without modeling and other treatments.

3.9. ERG Measurement. After that, the standard flash stimulation was used to induce retinal comprehensive potential (single stimulus). Dark ERG, bright ERG, max ERG, and $30 \mathrm{~Hz}$ flicker ERG were measured by the Roland visual electrophysiology diagnostic instrument system (Roland Industrial Electronics. L. P, Germany), and the frequency band was $0.1-1 \sim 300 \sim 1000 \mathrm{~Hz}$. During the experiment, animal warming equipment was used to maintain the body temperature of the animals. The ERG value measured in the blank control group was compared with the values measured in the model group and the drug administration group, respectively, to verify the effectiveness of the drug $[30,31]$.

3.10. Number of Retinal Pigment Epithelial Cells (RPE Cells) (Histopathological Examination). The left eyeballs of rats were fixed in $4 \%$ paraformaldehyde for two hours. The anterior segment and partial vitreous body were carefully removed. The remaining tissues were microscopically 
TABLE 1: The compositions of the Chuanqi microemulsion in situ gel and Chuanqi in situ gel.

\begin{tabular}{|c|c|c|c|c|}
\hline No. & Composition & $\begin{array}{l}\text { Chuanqi microemulsion in situ gel } \\
\qquad(\% \mathrm{w} / \mathrm{w})\end{array}$ & $\begin{array}{l}\text { Chuanqi in situ gel } \\
\qquad(\% \mathrm{w} / \mathrm{w})\end{array}$ & Remarks \\
\hline 1 & Chuanqi ethanol extract & 2.27 & 2.27 & \multirow{3}{*}{ Extract part } \\
\hline 2 & Chuanqi water extract & 2.72 & 2.72 & \\
\hline 3 & Oleic acid $(\mathrm{OA})$ & 0.08 & - & \\
\hline 4 & $\begin{array}{l}\text { Polyoxyethylene hydrogenated castor oil } \\
\qquad(\mathrm{RH}-40)\end{array}$ & 0.54 & - & \multirow{4}{*}{$\begin{array}{c}\text { Blank microemulsion } \\
\text { part }\end{array}$} \\
\hline 5 & Polyethylene glycol 400 (PEG 400) & 0.12 & - & \\
\hline 6 & Propylene glycol (PG) & 0.01 & - & \\
\hline 7 & Water (microemulsion part) & 1.52 & - & \\
\hline 8 & Poloxamer 407 & 17.00 & 17.00 & \multirow{3}{*}{ Blank in situ gel part } \\
\hline 9 & Gelatin & 0.17 & 0.17 & \\
\hline 10 & Water (in situ gel part) & 75.57 & 77.84 & \\
\hline
\end{tabular}

observed after dehydration, paraffin embedding, sectioning $(4 \mu \mathrm{m})$, and $\mathrm{HE}$ staining. The retinal pigment epithelia were counted using the DM2500 microscope with Image-Pro Plus 6.0 image analysis software (Leica Microsystems Inc., Route Planner, United States), the objective lens 40 times and the eyepiece 10 times were used for microscopic observation.

\subsection{Irritation [32-34]}

3.11.1. Specific Scoring Criteria. Each rabbit was scored according to the Technical Guideline Principles for Irritation and Hemolysis of Traditional Chinese Medicine and Natural Drugs (ZGPT4-1) (Center For Drug Evaluation) [34]; the irritation score between 0 and 3 was considered as no irritation, as shown in Table 2 .

3.11.2. One-Time Trial. After the ophthalmologic examinations, 30 male domestic rabbits $(3.0 \pm 0.5 \mathrm{~kg})$ with conforming eyes were selected, and they were randomly divided into three groups: Chuanqi microemulsion in situ gel group, gel matrix group, and normal saline group. The samples were dripped into the left eyes of the rabbits, and the eyes were examined (conjunctiva, cornea, iris, and secretions) at 1,3 , 24 , and 72 hours after the initiation of administration. The scores and photographs were recorded, and the two eyes of the same rabbit were compared.

3.11.3. Seven-Day Trial. After the ophthalmologic examinations, 30 male domestic rabbits $(3 \pm 0.5 \mathrm{~kg})$ with conforming eyes were selected, and then, they were randomly divided into three groups: Chuanqi microemulsion in situ gel group, gel matrix group, and normal saline group. The samples were dripped into the left eye of a male domestic rabbit four times per day (the administration time points were 9:00, 11:00, 13:00, and 15:00) for seven days. The eyes were examined for seven consecutive days. After 7 days, ophthalmological examinations were performed on rabbits, and the inspection items included conjunctiva, cornea, iris, and secretions. The scores and photographs were recorded, and the two eyes of the same rabbit were compared before and after administration.
TABLE 2: Criterion of irritation scores according to ZGPT4-1.

\begin{tabular}{lc}
\hline Irritation scores & Criterion \\
\hline $0 \sim 3$ & No irritation \\
$4 \sim 8$ & Low irritation \\
$9 \sim 12$ & Medium irritation \\
$13 \sim 16$ & High irritation \\
\hline
\end{tabular}

3.12. Tissue Distribution. There were two routes of drug absorption in the eye: corneal and noncorneal pathways, and the main route was the corneal pathway [35-38]. In this part, the tissue distribution of the Chuanqi microemulsion in situ gel was invested to discuss the absorption difference of index ingredients between the model animal and the normal animal. Furthermore, according to the results, the possible route across to the retina was speculated.

3.12.1. Collection of Tissue Samples. The corneas, lenses, vitreous bodies, and retinas of the rats' eyes were dissected successively. The collected tissues were transferred to the $1 \mathrm{~mL}$ centrifuge tubes and stored at $-80^{\circ} \mathrm{C}$.

3.12.2. Treatment of Tissue Distribution Samples. An appropriate amount of ocular tissues was homogenized, and then, the sample was extracted by ultrasound after adding $50 \%$ methanol-water. The above extracting solution was centrifuged at the rate of 13,200 rpm for 10 minutes, and the supernatant was analyzed by liquid chromatography-mass spectrometry.

\subsubsection{Liquid Chromatography-Mass Spectrometry} Conditions. Ligustrazine and ligustilide were the representative effective components of Ligusticum chuanxiong, and astragaloside IV was the representative effective component of Astragalus membranaceus (Figure 1). In this study, these components were used as the index ingredients to study the tissue distribution in vivo. The quantitative determination of ligustrazine, ligustilide, and astragaloside IV was performed by liquid chromatography-mass spectrometry (LC-MS). LC-20-AD rapid separation (Shimadzu Co., Ltd., Kyoto, Japan) and 5500 TRAP triple quadrupole 


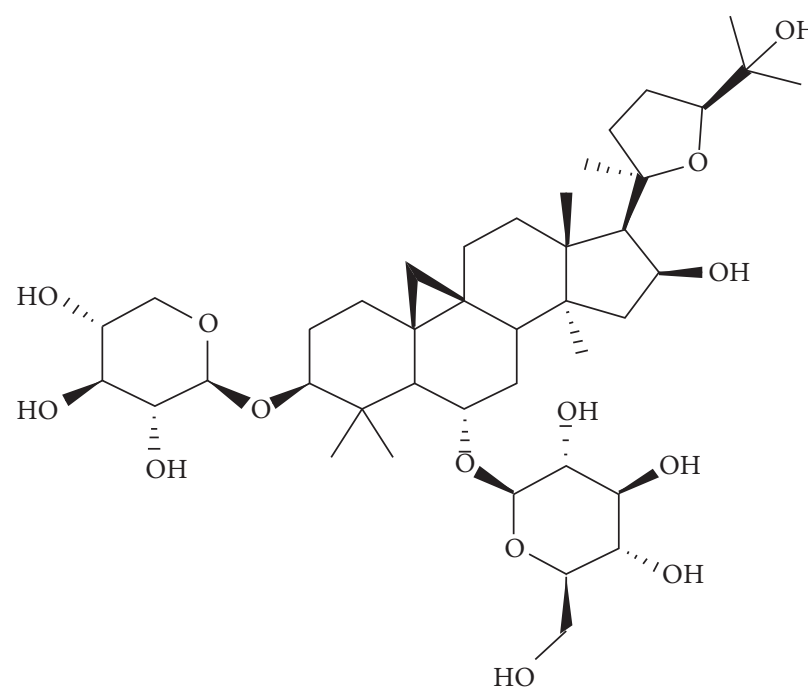

(a)

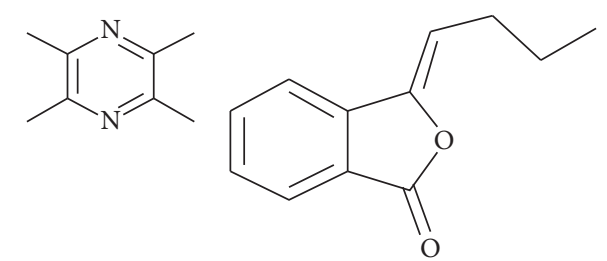

(b) (c)

FIgURE 1: The chemical structures of ligustrazine, ligustilide, and astragaloside IV.

tandem mass spectrometer (AB Scientific, Eltham, Australia) were used.

UPLC conditions were as follows: (1) column: Waters UPLC BEH C ${ }_{18}$ column $(1.7 \mu \mathrm{m}, 2.1 \mathrm{~mm} \times 100 \mathrm{~mm})$; column temperature: $40^{\circ} \mathrm{C}$; mobile phase: water $(0.1 \%$ formic acid $)$ and acetonitrile; and flow rate: $0.3 \mathrm{~mL} \cdot \mathrm{min}^{-1}$, and gradient elution program is shown in Table 3 . Every sample was filtered through a $0.22 \mu \mathrm{m}$ membrane filter before injection into the UPLC system. The analytes were detected with electrospray positive ionization $\left(\mathrm{ESI}^{+}\right)$. The ion pairs and collision energies of the analytes were as follows: ligustilide: $(\mathrm{m} / \mathrm{z}: \quad 191.1 \longrightarrow 91.1, \quad 15 \mathrm{~V}), \quad$ astragaloside IV $(\mathrm{m} / \mathrm{z}$ : $785.5 \longrightarrow 143.1,25 \mathrm{~V})$, and ligustrazine $(\mathrm{m} / \mathrm{z}: 137.1 \longrightarrow 96.1$, $20 \mathrm{~V}$ ), and other details are shown in Table 4.

\subsubsection{Tissue Distribution Study}

(1) The Local Tissue Distribution of the Chuanqi Microemulsion In Situ Gel in the Dry-AMD Model Animals. Male SD rats $(160 \mathrm{~g} \pm 10 \mathrm{~g})$ were divided into two groups: Chuanqi microemulsion in situ gel group (drug concentration: 5\%) and blank group (normal saline) with 16 rats in each group. The SD rats in the Chuanqi microemulsion in situ gel modeling group were treated according to the method under Section "3.8. Model Establishment and Grouping" to establish the dry-AMD models. The Chuanqi microemulsion in situ gel was administrated after the modeling was completed. The administration methods and sampling points of each group were as follows: $20 \mu \mathrm{L}$ of the sample was dripped into the two eyes of male SD rats; the rats were sacrificed at $0.5,1,2$, and 4 hours after administration, and then the two eyeballs of SD rats were stripped (4 SD rats at each sampling point). The ocular tissues were treated and determined with the method mentioned in Section "3.12 Tissue Distribution, (2) Treatment of Tissue Distribution Samples."
TABLE 3: Gradient elution program of LC-MS.

\begin{tabular}{lcc}
\hline Time $(\min )$ & Water $(v \%)$ & Acetonitrile $(v \%)$ \\
\hline 0 & 95 & 5 \\
2 & 50 & 50 \\
4 & 20 & 80 \\
5 & 0 & 100 \\
6 & 0 & 100 \\
6.1 & 95 & 5 \\
10 & 95 & 5 \\
\hline
\end{tabular}

TABLE 4: Parameters of mass spectrometry.

\begin{tabular}{lcc}
\hline CMR: 30.00 & CAD: medium & CXP $: 10.00$ \\
\hline IS $: 5000.00$ & DP $: 120.0$ & GS1 $: 50.00$ \\
TEM $: 500.00$ & EP $: 10.0$ & GS2 $: 50.00$ \\
\hline
\end{tabular}

(2) A Comparative Study of the Local Tissue Distribution between the Chuanqi Microemulsion In Situ Gel and Chuanqi In Situ Gel in the Normal Animal [39]. Male SD rats $(160 \mathrm{~g} \pm 10 \mathrm{~g})$ were divided into three groups: Chuanqi microemulsion in situ gel group (drug concentration: 5\%), Chuanqi in situ gel group (drug concentration: 5\%), and blank group (normal saline), with 16 rats in each group; the research method is shown in [39].

3.13. Statistical Analysis. The results in the article were statistically analyzed by means of SPSS 19.0. Multiple randomized single-factor analysis of variance (ANOVA) was used to statisticize the data between multiple groups at the same time point. According to the results of the homogeneity of variance test, the statistical methods of comparing each other were determined, $p>0.05$, LSD method was used; $p<0.05$, and Tamhane method was used; $p<0.05$ was considered statistically significant. 


\section{Results}

4.1. Characterization. The characterizations of the Chuanqi microemulsion in situ gel were investigated using the method given under Section " 3.4 Characterizations of Chuanqi Microemulsion In Situ Gel." The result showed that the particle size of microemulsion was $38.56 \pm 0.21 \mathrm{~nm}$ (PDI 0.18 ), and the zeta potential was $-17.99 \pm 0.22 \mathrm{mv}$.

4.2. Morphological Observation of Microemulsion. The morphology of Chuanqi microemulsion was observed using the method given under Section "3.5 Morphological Observation of Microemulsion," and the pictures are shown in Figure 2; the particle size of the microemulsion was shown within $100 \mathrm{~nm}$, and it was unevenly globular or quasiglobular.

\subsection{The Phase Transition Temperature of the Chuanqi} Microemulsion In Situ Gel. The phase transition temperature of the Chuanqi microemulsion in situ gel was $32 \pm 0.5^{\circ} \mathrm{C}$, which was basically the same as that of the cornea at $32.06 \pm 1.24^{\circ} \mathrm{C}$ [40]. Therefore, the in situ gel could be changed from liquid to semisolid in the cornea.

\subsection{Evaluation of Efficacy Based on the Dry-AMD Animal Model}

4.4.1. Dark ERG (Rod Cells). The results of dark adaptation ERG showed that the $b$-wave amplitude is arranged in the descending order: esculin and digitalis glycosides eye drops group $>$ Chuanqi microemulsion in situ gel group $>$ normal control group $>$ model control group. The amplitude of the $b$-wave in each group had statistical significance $(p<0.05)$. The amplitude of the normal control group, Chuanqi microemulsion in situ gel group, and esculin and digitalis glycosides eye drops group was significantly higher than that of the model group $(p<0.05)$, as shown in Table 5 and Figures 3 and 4 . The result of dark ERG showed that the Chuanqi microemulsion in situ gel had a protective effect on the rod cells.

4.4.2. Max ERG. The results of dark adaptation max ERG showed that the $b$-wave amplitude is arranged in the descending order: Chuanqi microemulsion in situ gel group $>$ normal control group $>$ esculin and digitalis glycosides eye drops group $>$ model control group. The amplitude of the $b$-wave in each group had statistical significance $(p<0.05)$. The amplitude of the normal control group, Chuanqi microemulsion in situ gel group, and esculin and digitalis glycosides eye drops group was significantly higher than that of the model group $(p<0.05)$, as shown in Table 6 and Figures 3 and 5.

4.4.3. Bright ERG (Cone Cells). The results of bright adaptation ERG showed that the $b$-wave amplitude is arranged in the descending order: Chuanqi microemulsion in situ gel group $>$ esculin and digitalis glycosides eye drops

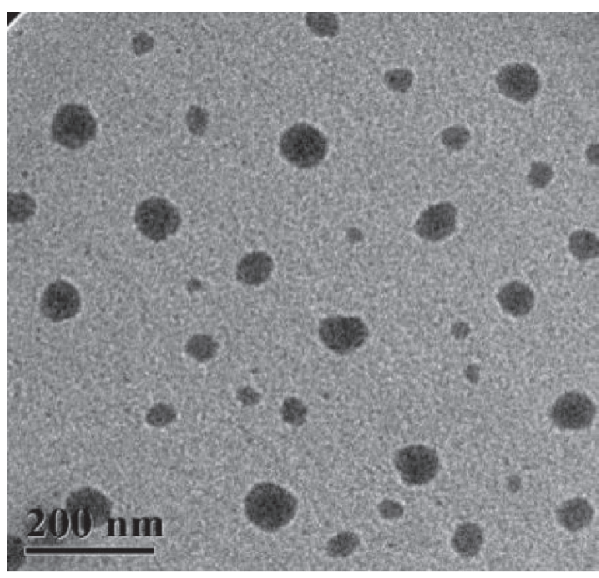

Figure 2: TEM of Chuanqi microemulsion.

group $>$ normal control group $>$ model control group. The amplitude of the $b$-wave in each group had statistical significance $(p<0.05)$. The amplitude of the normal control group, Chuanqi microemulsion in situ gel group, and esculin and digitalis glycosides eye drops group was significantly higher than that of the model group $(p<0.05)$, as shown in Table 7 and Figures 3 and 6. The result of bright ERG showed that the Chuanqi microemulsion in situ gel had a protective effect on the cone cells.

4.4.4. $30 \mathrm{~Hz}$ Flicker ERG. The results of bright adaptation $30 \mathrm{~Hz}$ flicker ERG showed that the $b$-wave amplitude is arranged in the descending order: Chuanqi microemulsion in situ gel group $>$ esculin and digitalis glycosides eye drops group $>$ normal control group $>$ model control group. The amplitude of the $b$-wave in each group had statistical significance $(p<0.05)$. The amplitude of the normal control group, Chuanqi microemulsion in situ gel group, and esculin and digitalis glycosides eye drops group was significantly higher than that of the model group $(p<0.05)$, as shown in Table 8 and Figures 3 and 7 .

4.4.5. Number of Retinal Pigment Epithelial Cells (RPE Cells). The result of RPE cell count showed that the order of RPE cell count was as follows: normal control group $>$ Chuanqi microemulsion in situ gel group $>$ esculin and digitalis glycosides eye drops group $>$ model control group. The number of RPE cells in each group was statistically significant $(p<0.05)$. The results are shown in Table 9 and Figure 8 .

4.5. Irritation. The irritation of the Chuanqi microemulsion in situ gel was evaluated through one-time trial and 7-day trial, with the specific methods shown under Section "Irritation". It turned out that, at $3 \mathrm{~h}$ after the one-time administration test, the irritation scores of the Chuanqi microemulsion in situ gel group, matrix group, and saline group were $0.05,0$, and 0 , respectively, as shown in Table 10 .

The irritation scores of the Chuanqi microemulsion in situ gel group, matrix group, and saline group in 7-day dose 
TABLE 5: The results of dark ERG.

\begin{tabular}{lcc}
\hline Group & Number of samples & Values \\
\hline Normal control group & 11 & $30.73^{*} \pm 8.25$ \\
Model control group & 8 & $11.00 \pm 2.51$ \\
Esculin and digitalis glycosides eye drops group & 13 & $38.92^{*} \pm 7.37$ \\
Chuanqi microemulsion in situ gel & 11 & $37.23^{*} \pm 5.44$ \\
Total & 43 & \\
\hline
\end{tabular}

*Statistical significance $(p<0.05)$.

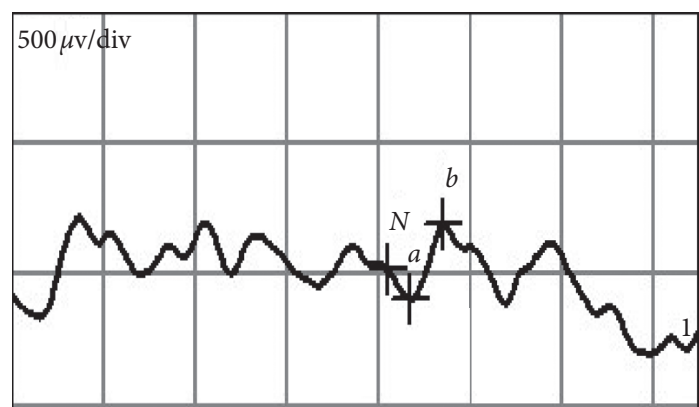

(a)

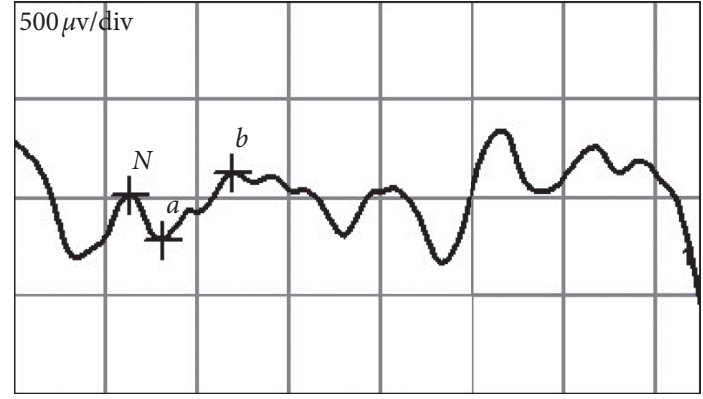

(c)

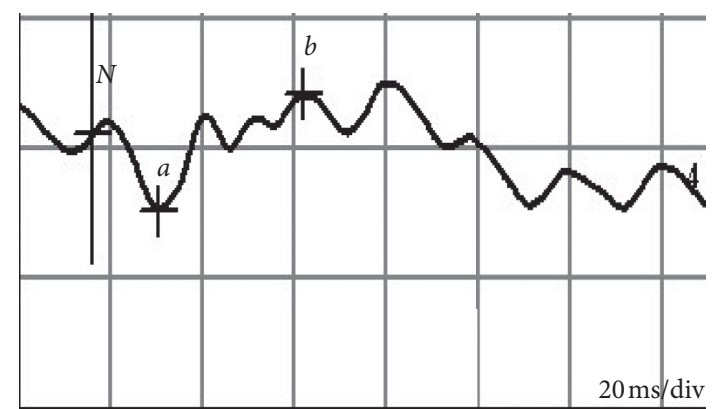

(b)

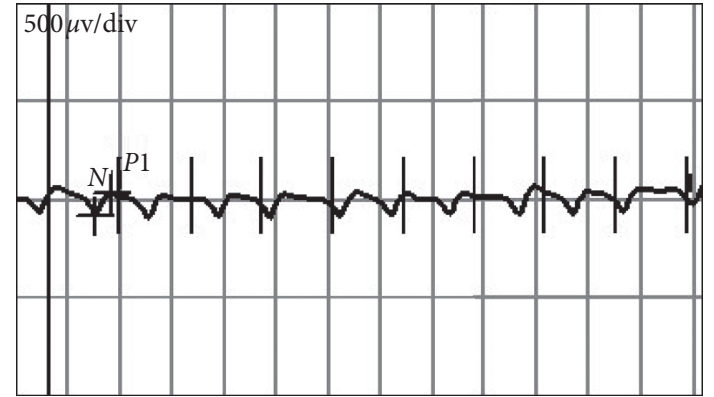

(d)

Figure 3: Electroretinograms of the efficacy evaluations: (a) dark ERG; (b) max ERG; (c) bright ERG; (d) 30 Hz flicker ERG.

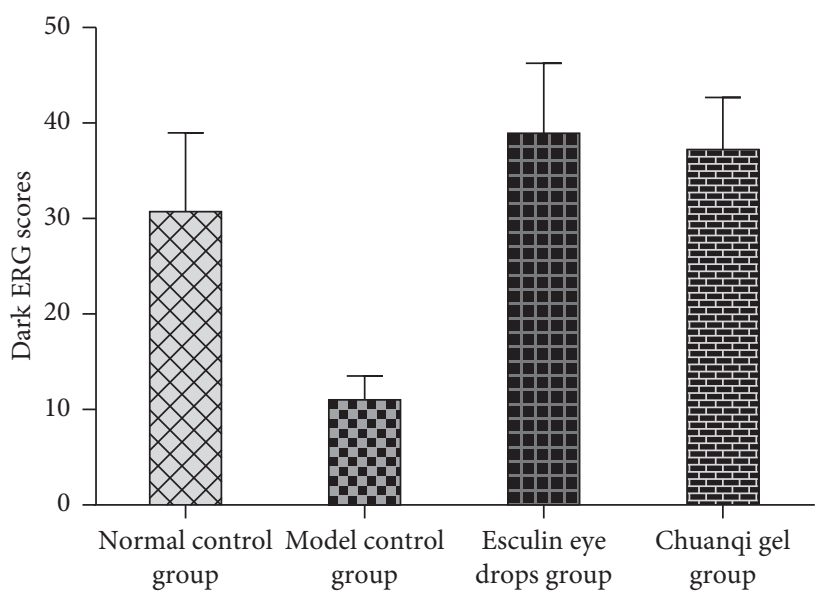

Figure 4: Scores of dark ERG (Chuanqi microemulsion in situ gel is abbreviated to Chuanqi gel; esculin and digitalis glycosides eye drops group is abbreviated to esculin eye drops group).

trials were 1.0, 0.06, and 0, respectively, as shown in Figure 9. Rabbit eyes of the Chuanqi microemulsion in situ gel group, matrix group, and saline group were almost the same.
According to the irritant scoring criteria, 0-3 were classified as no irritation (Table 10) [1]. It turned out that the Chuanqi microemulsion in situ gel had no irritation.

\subsection{Tissue Distribution}

4.6.1. In Vivo Absorption Characteristics of the Chuanqi Microemulsion In Situ Gel. Microemulsion could increase the absorption of the drug in the eye of normal animals; the research [39] showed that, in the Chuanqi in situ gel administration group, a very small amount of ligustilide $(24.31 \pm 5.21 \mathrm{ng} / \mathrm{g})$ was only detected in the cornea at the 30 min sampling time point (Figures 10 and 11), and no any indicative component was detected in the vitreous body, retina, and crystalline lens at all sampling time points; in the Chuanqi microemulsion in situ gel administration group, ligustilide could be detected in different eye tissues at different sampling time points. The results showed that the drug carriers of microemulsion could markedly improve the ocular penetration and enhance the absorption of the drug.

As the research deepened, the local tissue distribution study on the Chuanqi microemulsion in situ gel in the 
TABle 6: The results of max ERG.

\begin{tabular}{lcc}
\hline Group & Number of samples & Values \\
\hline Normal control group & 10 & $26.30^{*} \pm 3.28$ \\
Model control group & 8 & $12.00 \pm 1.00$ \\
Esculin and digitalis glycosides eye drops group & 10 & $25.50^{*} \pm 3.74$ \\
Chuanqi microemulsion in situ gel & 10 & $38.90^{*} \pm 4.15$ \\
Total & 38 & \\
\hline
\end{tabular}

${ }^{*}$ Statistical significance $(p<0.05)$.

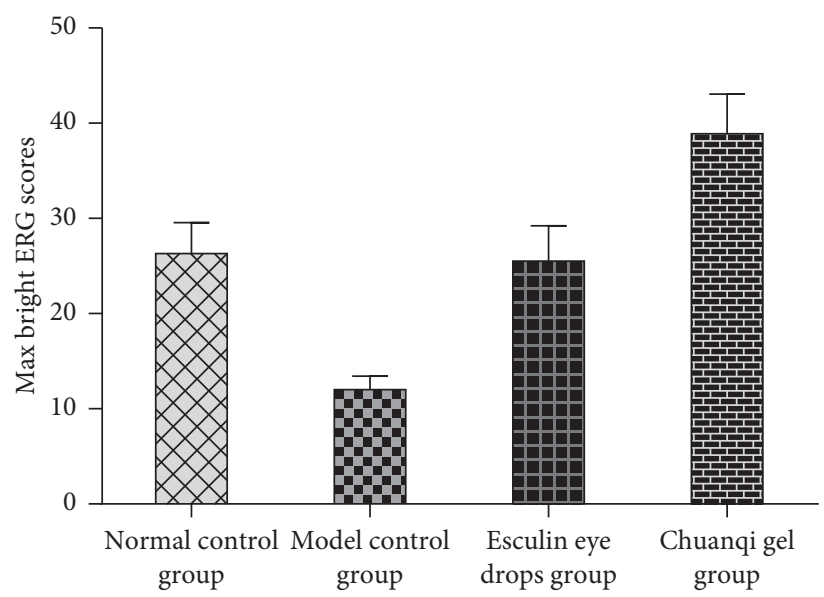

FIgURE 5: Scores of max ERG (Chuanqi microemulsion in situ gel is abbreviated to Chuanqi gel; esculin and digitalis glycosides eye drops group is abbreviated to esculin eye drops group).

TABLE 7: The results of bright ERG.

\begin{tabular}{lcc}
\hline Group & Number of samples & Values \\
\hline Normal control group & 6 & $18.83^{*} \pm 5.25$ \\
Model control group & 6 & $6.58 \pm 1.46$ \\
Esculin and digitalis glycosides eye drops group & 12 & $22.17^{*} \pm 7.53$ \\
Chuanqi microemulsion in situ gel & 8 & $36.13^{*} \pm 9.22$ \\
Total & 32 & \\
\hline
\end{tabular}

${ }^{*}$ Statistical significance $(p<0.05)$.

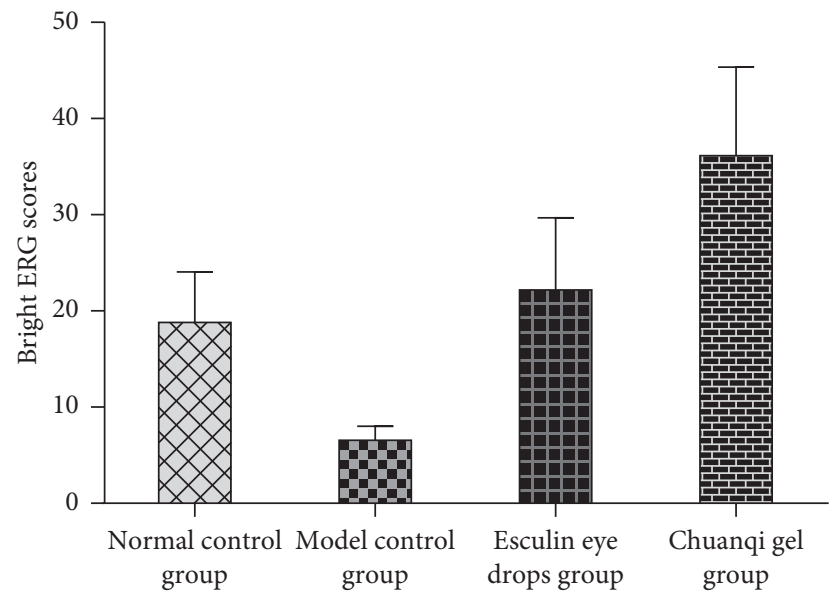

FIgURE 6: Scores of bright ERG (Chuanqi microemulsion in situ gel is abbreviated to Chuanqi gel; esculin and digitalis glycosides eye drops group is abbreviated to esculin eye drops group.). 
TABLE 8: The results of flicker ERG.

\begin{tabular}{lcc}
\hline Group & Number of samples & Values \\
\hline Normal control group & 10 & $14.85^{*} \pm 1.20$ \\
Model control group & 6 & $6.50 \pm 0.74$ \\
Esculin and digitalis glycosides eye drops group & 7 & $21.29^{*} \pm 2.16$ \\
Chuanqi microemulsion in situ gel & 8 & $32.44^{*} \pm 2.87$ \\
Total & 31 & \\
\hline
\end{tabular}

*Statistical significance $(p<0.05)$.

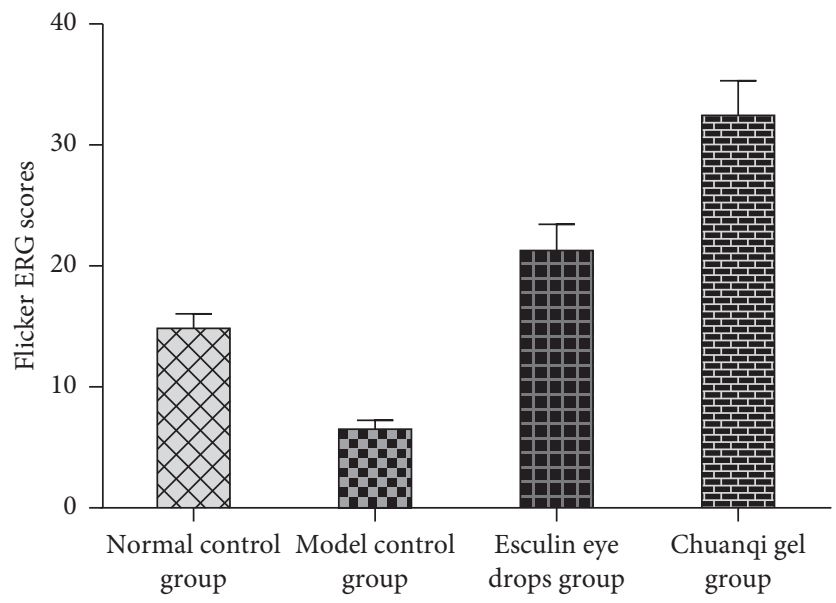

Figure 7: Scores of $30 \mathrm{~Hz}$ flicker ERG (Chuanqi microemulsion in situ gel is abbreviated to Chuanqi gel; esculin and digitalis glycosides eye drops group is abbreviated to esculin eye drops group).

TABLE 9: Statistical table of the number of RPE cells.

\begin{tabular}{lcc}
\hline Group & Number of samples & Values \\
\hline Normal control group & 8 & $48.31 \pm 5.74$ \\
Model control group & 16 & $11.41 \pm 0.88$ \\
Esculin and digitalis glycosides eye drops group & 11 & $31.55^{*} \pm 2.58$ \\
Chuanqi microemulsion in situ gel & 11 & $36.45^{*} \pm 1.74$ \\
Total & 46 & \\
\hline
\end{tabular}

* Statistical significance $(p<0.05)$.

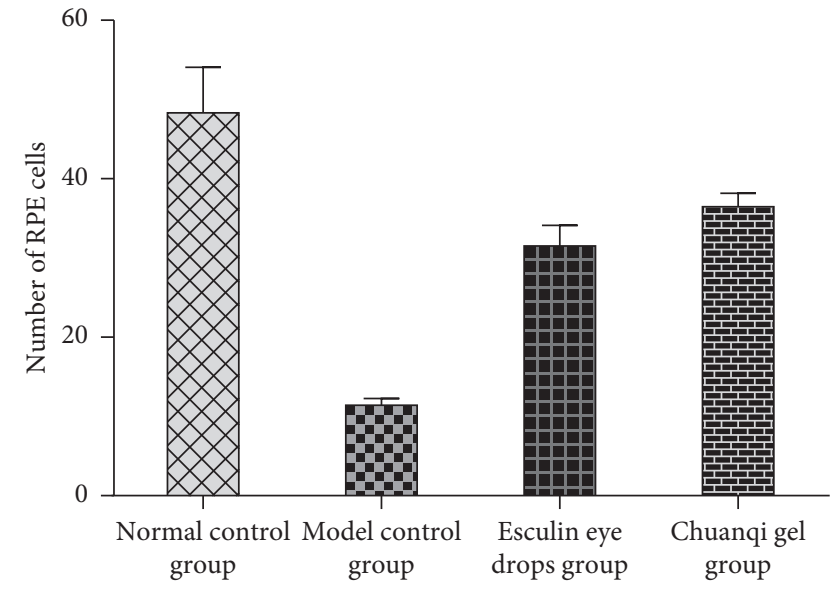

Figure 8: The result of RPE cell count (Chuanqi microemulsion in situ gel is abbreviated to Chuanqi gel; esculin and digitalis glycosides eye drops group is abbreviated to esculin eye drops group).
dry-AMD model animals was carried out. The process of the tissue distribution of the Chuanqi microemulsion in situ gel was discussed, and the possible route across to the retina was speculated.

The results showed that only ligustrazine could be quantitatively determined at different time points, while ligustrazine and astragaloside IV cannot be determined (Tables 11 and 12, Figure 12). In addition, compared with the normal group, the intraocular absorption of ligustrazine in the AMD model group increased significantly; at the $30 \mathrm{~min}$ sampling point, the contents of ligustrazine in the cornea, vitreous body, and retina increased by $23.7 \%, 21.2 \%$, and $29.4 \%$, respectively; the decline of the eye barrier in the AMD model group may be the cause of this phenomenon.

4.6.2. The Possible Route across to the Retina of Ligustilide from the Chuanqi Microemulsion In Situ Gel. The possible route across to the retina was speculated as follows. At 30 
TABLe 10: Statistical results of irritation scores at one dose trial $(n=10)$.

\begin{tabular}{|c|c|c|c|c|}
\hline \multirow{2}{*}{ Group } & \multicolumn{4}{|c|}{ Irritation scores } \\
\hline & $1 \mathrm{~h}$ & $3 \mathrm{~h}$ & $24 \mathrm{~h}$ & $72 \mathrm{~h}$ \\
\hline Chuanqi group & 0 & $0.05 \pm 0.16$ & 0 & 0 \\
\hline Matrix group & 0 & 0 & 0 & 0 \\
\hline Saline group & 0 & 0 & 0 & 0 \\
\hline
\end{tabular}

Chuanqi microemulsion in situ gel group is abbreviated to Chuanqi group.

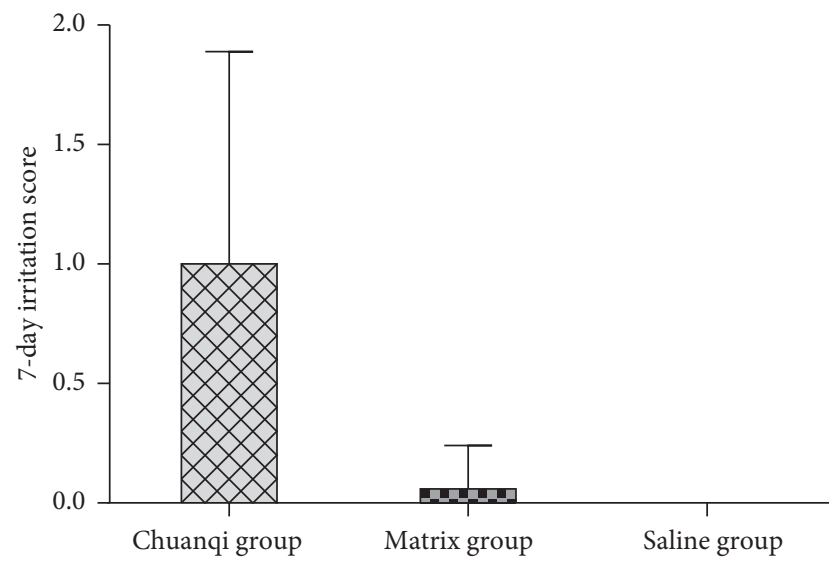

Figure 9: Irritation scores in 7-day dose trials (Chuanqi microemulsion in situ gel group is abbreviated to Chuanqi group; esculin and digitalis glycosides eye drops group is abbreviated to esculin eye drops group).

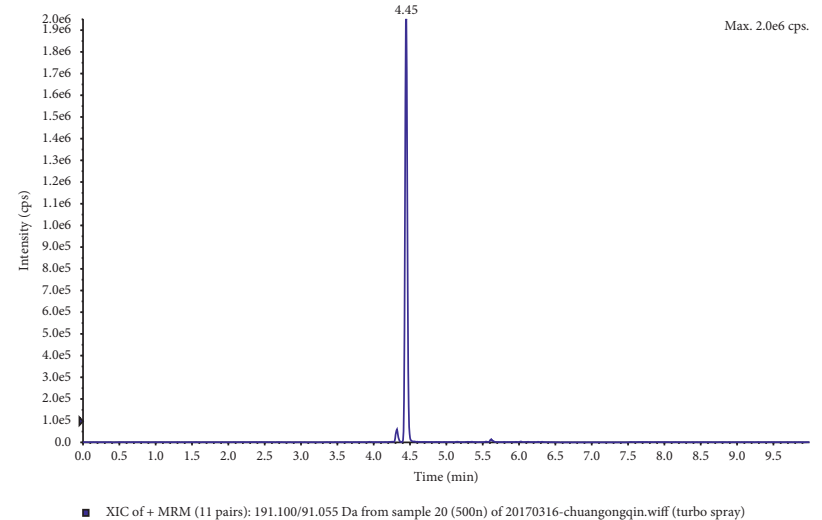

(a)

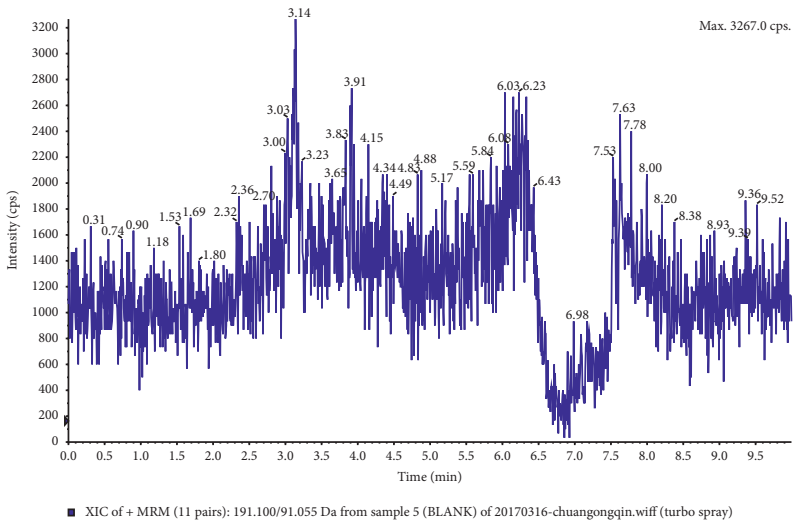

(b)

Figure 10: (a) LC-MS chromatogram of ligustilide; (b) blank LC-MS chromatogram of ligustilide.

minutes, ligustilide had reached the vitreous body and retina, and its concentrations in the cornea, vitreous body, and retina were $293.88 \pm 21.43 \mathrm{ng} / \mathrm{g}, 106.98 \pm 8.67 \mathrm{ng} / \mathrm{g}$, and $76.36 \pm 5.88 \mathrm{ng} / \mathrm{g}$, respectively. The concentration of ligustilide in these three ocular structures decreased with time. Four hours after administration, ligustilide was still present in the cornea, but it could not be detected in the vitreous body and retina.

To sum up, the concentration order of ligustilide in the eye tissue was as follows: cornea $>$ vitreous body $>$ retina. In terms of the eyeball structure, the cornea was at the anterior side of the eyeball, the vitreous body was located at the middle-posterior side of the eyeball, and the retina was located at the posterior side of the eyeball. The concentration order of ligustilide in the cornea, vitreous body, and retina was related to the eye structural features. The drug absorption process was passive diffusion. In this sense, it was concluded that the cornea-vitreous body-retina was one of the routes for the drug to penetrate from the anterior region to the posterior region.

The Chuanqi microemulsion in situ gel was used for the treatment of dry-AMD, which was a posterior ocular disease. The distribution results, obtained from the normal animals and the dry-AMD model animals, showed that the active component in the Chuanqi microemulsion in situ gel could exert effects after reaching the posterior eyes. 


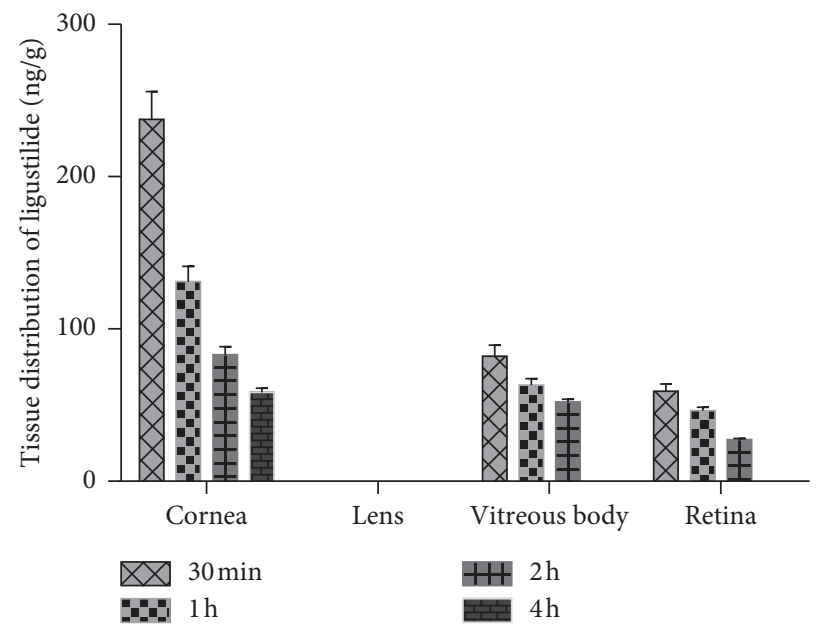

FIgURE 11: Tissue distribution of ligustilide in the Chuanqi microemulsion in situ gel (normal animals).

TABle 11: The distribution of ligustilide (dry-AMD animals, microemulsion gel group, $n=4$ ).

\begin{tabular}{lcccc}
\hline Time points/tissues & Cornea $(\mathrm{ng} / \mathrm{g})$ & Lens $(\mathrm{ng} / \mathrm{g})$ & Vitreous body $(\mathrm{ng} / \mathrm{g})$ & Retina $(\mathrm{ng} / \mathrm{g})$ \\
\hline $30 \mathrm{~min}$ & $293.88 \pm 21.43$ & 0 & $106.98 \pm 8.67$ & $76.36 \pm 5.88$ \\
$1 \mathrm{~h}$ & $164.07 \pm 12.18$ & 0 & $85.58 \pm 5.42$ & $53.45 \pm 3.63$ \\
$2 \mathrm{~h}$ & $103.51 \pm 7.10$ & 0 & $68.46 \pm 3.21$ & $37.42 \pm 1.71$ \\
$4 \mathrm{~h}$ & $77.42 \pm 3.62$ & 0 & 0 & 0 \\
\hline
\end{tabular}

TABLE 12: The distribution of ligustilide (dry-AMD animals, blank group, $n=4$ ).

\begin{tabular}{lcccc}
\hline Time points/tissues & Cornea $(\mathrm{ng} / \mathrm{g})$ & Lens $(\mathrm{ng} / \mathrm{g})$ & Vitreous body $(\mathrm{ng} / \mathrm{g})$ & Retina $(\mathrm{ng} / \mathrm{g})$ \\
\hline $30 \mathrm{~min}$ & 0 & 0 & 0 & 0 \\
$1 \mathrm{~h}$ & 0 & 0 & 0 & 0 \\
$\mathrm{~h}$ & 0 & 0 & 0 & 0 \\
$4 \mathrm{~h}$ & 0 & 0 & 0 \\
\hline
\end{tabular}

\section{Discussion}

In this paper, Chuanqi microemulsion was prepared with OA, PEG400, and PG; the particle size of the microemulsion was less than $100 \mathrm{~nm}$, and its appearance was spherical. Besides, Chuanqi microemulsion in situ gel was prepared by the combination of Chuanqi microemulsion and in situ gel. The nanopreparation changed from liquid to semisolid after instilling into the eye, and the drug could reside in the eyes for a longer period of time. With the help of microemulsion, the small molecule compound could quickly penetrate the cornea and lens to reach the ocular posterior segment, which would be beneficial to dry-AMD treatment. This article systematically evaluated the novel ophthalmic preparations from the perspectives of efficacy, irritation, and tissue distribution.

5.1. Discussion on Pharmacodynamic Results. The retinal tissue of mammals generally contained two types of photoreceptor cells: rod cells and cone cells. Dark adaptation ERG and max ERG could be used to evaluate the physiological function of rod cells; bright adaptation ERG and

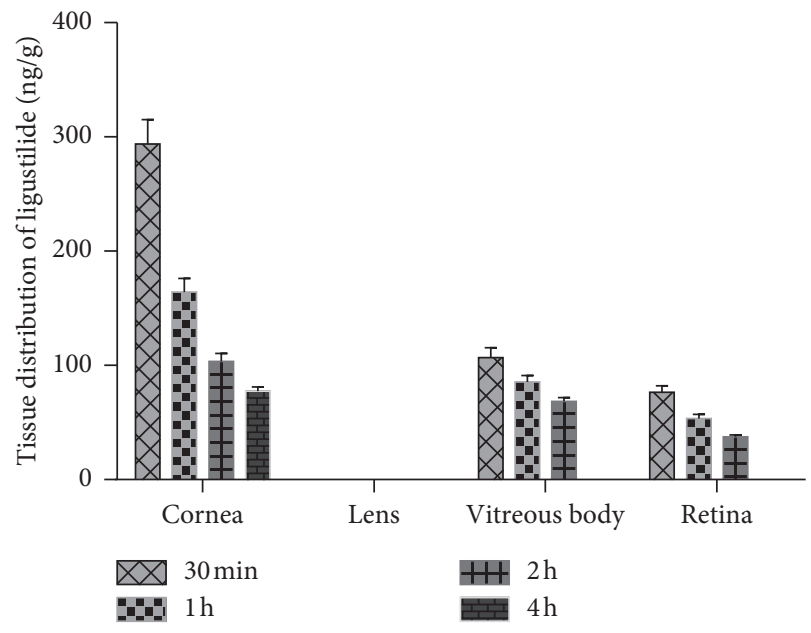

FIgUre 12: Tissue distribution of ligustilide in the Chuanqi microemulsion in situ gel (dry-AMD animals).

$30 \mathrm{~Hz}$ flicker ERG could be used to evaluate the physiological function of cone cells, and $30 \mathrm{~Hz}$ flicker ERG could also evaluate the blood circulation in the retina [41-43]. Retinal 
pigment epithelial cells could support, nourish, and protect the photoreceptor cells, and the number of these cells could show the function and state of the retina [44]. This study showed that compared with other drugs, the Chuanqi microemulsion in situ gel had significantly improved $b$-wave amplitudes of dark ERG, dark max ERG, light ERG, and flicker ERG in the dry-AMD model animals. These results of ERG and retinal pigment epithelial cell count indicated that the Chuanqi microemulsion in situ gel had obvious advantages in improving the function of photoreceptor cells and improving blood circulation in the retina. That is to say the novel ophthalmic nanopreparation can reduce the symptoms of dry-AMD in animals.

5.2. Discussion on the Results of Tissue Distribution Research. Compared with the Chuanqi microemulsion in situ gel and Chuanqi in situ gel, the index ingredient (ligustrazine) could reach the posterior eye segment faster. Ligustrazine in the Chuanqi microemulsion in situ gel could reach the retina through the cornea and vitreous body within 30 minutes in normal and AMD model rats. In addition, microemulsion in situ gel could deliver drugs to eye tissues of the AMD model rat more efficiently than the normal rat; the decline of the eye barrier in the AMD model rat might be the cause of this phenomenon. In the following study, the drug eye permeation route of ligustrazine in the Chuanqi microemulsion in situ gel was studied, and results showed that ligustilide could penetrate from the anterior region to the posterior region through the route of cornea-vitreous body-retina. The sequence of the ligustilide concentration was cornea $>$ vitreous body $>$ retina. The relevant research provided a basis for understanding the mechanism and law of eye penetration.

In this study, astragaloside IV and ligustilide were not detected in the cornea, lens, and retina of each animal. The possible reasons for this phenomenon were as follows: (1) astragaloside IV had a large molecular weight and strong polarity, which were not conducive to crossing the biological barrier; in addition, the drug-metabolizing enzyme in the eye made the drug transformed and could not be detected; (2) ligustilide was less stable, and it was more easily degraded by metabolic enzymes in the eye. Although the above two components had not been quantitatively measured, their possible metabolites in the eye might also have a therapeutic effect.

To sum up, the combination of TCM and nanotechnology could increase the concentration of the active ingredients of TCM in the posterior segment of the eye, thereby improving the therapeutic effect of TCM on the ocular posterior segment eye disease. The attained results from this study could provide a platform for the development of new therapeutic drugs for other posterior segment eye diseases, such as diabetic retinopathy, glaucoma, and retinal pigment degeneration.

\section{Conclusion}

The particle size of the Chuanqi microemulsion was $38.56 \pm 0.21 \mathrm{~nm}$, and it was unevenly globular or quasiglobular. The results of pharmacodynamic studies showed that the Chuanqi microemulsion in situ gel had certain roles in repairing retina damage of the dry-AMD animal model. The advantage of no irritation was beneficial to develop a nanopreparation for dry-AMD. The results of pharmacokinetic studies showed that the microemulsion in situ gel could effectively deliver drugs to the posterior eye of the AMD model rat through the route of cornea-vitreous bodyretina. This paper provided a meaningful research strategy and research basis for the development of new dry-AMD therapeutic drugs.

\section{Data Availability}

The data used to support the findings of this study are available from the corresponding author upon request.

\section{Disclosure}

Maobo Du and Shuo Shen are the co-first authors of this paper.

\section{Conflicts of Interest}

The authors report no conflicts of interest in this work.

\section{Authors' Contributions}

Maobo Du and Shuo Shen contributed equally to this work.

\section{Acknowledgments}

This work was supported by grants from the National Natural Science Foundation of China (Grant no. 81373977) and the Fundamental Research Funds for the Central Public Welfare Research Institutes (Grant nos. ZZ13-YQ-052 and ZZ13-YQ-108).

\section{References}

[1] World Health Organization, Vision 2020 the Right to Slight, World Health Organization, Geneva, Switzerland, 2020, http://www.who.int/blindness/Vision2020_report.pdf?ua=1.

[2] R. Kumar-Singh, "The role of complement membrane attack complex in dry and wet AMD-from hypothesis to clinical trials," Experimental Eye Research, vol. 184, pp. 266-277, 2019.

[3] M. B. Du, S. Z. Liu, K. Xu et al., "Summarize drug dosage forms in treatment of age-related macular degeneration disease," Chinese Journal Experiment Traditional Medicine Formulae, vol. 42, no. 4, pp. 628-633, 2017.

[4] S. Q. Tan, X. Geng, J. H. Liu et al., "Xue-fu-zhu-yu decoction protects rats against retinal ischemia by downregulation of HIF- $1 \alpha$ and VEGF via inhibition of RBP2 and PKM2," BMC Complementary and Alternative Medicine, vol. 17, p. 365, 2017.

[5] Y. M. Lee, Y. R. Lee, C. S. Kin et al., "Cnidium officinale extract and butylidenephthalide inhibits retinal neovascularization in vitro and in vivo," BMC Complementary and Alternative Medicine, vol. 16, p. 231, 2016.

[6] Chinese Pharmacopoeia Committee, Pharmacopoeia of the People's Republic of China 2015, China Medical Science Press, Beijing, China, 2015. 
[7] W. L. Shi, J. Zhao, R. Yuan et al., "Combination of Ligusticum chuanxiong and radix paeonia promotes angiogenesis in ischemic myocardium through notch signalling and mobilization of stem cells," Evidence-Based Complementary and Alternative Medicine, vol. 2019, Article ID 7912402, 12 pages, 2019.

[8] C. W. Li, W. Shen, and B. S. Wu, "Efficacy of Ligusticum chuanxiong in treating retinal vein occlusion," Chinese Journal of Practical Ophthalmology, vol. 20, no. 12, pp. 945-947, 2002.

[9] K. Q. Zhao and J. He, "Effect of Astragalus membranaceus on retina in rats with the damage of retinal ischemia-reperfusion," International Journal of Ophthalmology, vol. 6, no. 5, pp. 1042-1044, 2006.

[10] L. R. Yin and J. S. Gao, "Protective effects of Radix astragali on retinal ganglion cells of high intraocular pressure rats," Journal of Beijing University of Traditional Chinese Medicine, vol. 39, no. 10, pp. 828-832, 2016.

[11] G. Velez and S. M. Whitcup, "New developments in sustained release drug delivery for the treatment of intraocular disease," British Journal of Ophthalmology, vol. 83, no. 11, pp. 12251229, 1999.

[12] J. H. Schulman, "Transparent water-in-oil dispersions: the oleopathic hydro-micelle," Nature, vol. 152, no. 3847, pp. 102-103, 1943.

[13] Y. Q. Lin, Y. Gan, L. Gan et al., "Preparation of dexamethasone microemulsion for ocular application," Chinese Pharmaceutical Journal, vol. 41, no. 5, pp. 358-362, 2006.

[14] S. A. Tayel, M. A. El-Nabarawi, M. I. Tadros et al., "Promising ion-sensitive in situ ocular nanoemulsion gels of terbinafine hydrochloride: design, in vitro characterization and in vivo estimation of the ocular irritation and drug pharmacokinetics in the aqueous humor of rabbits," International Journal of Pharmaceutics, vol. 443, no. 1-2, pp. 293-305, 2013.

[15] J. J. Reidy, B. M. Gebhardt, and H. E. Kaufman, "The collagen shield: a new vehicle for delivery of cyclosporine to the eye," Cornea, vol. 9, pp. 196-199, 1990.

[16] A. H. El-Kamel, "In vitro and in vivo evaluation of pluronic F127-based ocular delivery system for timolol maleate," International Journal of Pharmaceutics, vol. 241, no. 1, pp. 47$55,2002$.

[17] H. Y. Wu, Z. D. Liu, J. J. Peng et al., "Design and evaluation of baicalin-containing in situ $\mathrm{pH}$-triggered hydrogelling system for sustained ophthalmic drug delivery," International Journal of Pharmaceutics, vol. 410, no. 1-2, pp. 31-40, 2011.

[18] M. C. Bonferoni, P. Chetoni, P. Giunchedi et al., "Carrageenan-gelatin mucoadhesive systems for ion-exchange based ophthalmic delivery: in vitro and preliminary in vivo studies," European Journal of Pharmaceutics and Biopharmaceutics, vol. 57, no. 3, pp. 465-472, 2004.

[19] J. R. Rossi and G. M. Mlynek, "Bioadhesive and phase-change polymers for ocular drug delivery," Advanced Drug Delivery Reviews, vol. 16, no. 1, pp. 45-50, 1995.

[20] A. Dalpiaz, A. Scatturin, F. Bortolotti et al., "Cationic liposomes as potential carriers for ocular administration of peptides with anti-herpetic activity," International Journal of Pharmaceutics, vol. 317, no. 1, pp. 90-100, 2006.

[21] M. E. Bosch, A. J. R. Sánchez, F. S. Rojas, and C. B. Ojeda, "Analytical methodologies for the determination of cisplatin," Journal of Pharmaceutical and Biomedical Analysis, vol. 47, no. 3, pp. 451-459, 2008.

[22] M. B. Ojeda, S. Shen, Q. Chen et al., "Screening of solid-type surfactants in microemulsions by particle size analysis," Chinese Journal Experiment Traditional Medicine Formulae, vol. 21, no. 22, pp. 137-139, 2015.
[23] Y. Jiang, F. Li, Y. Luan et al., "Formation of drug/surfactant catanionic vesicles and their application in sustained drug release," International Journal of Pharmaceutics, vol. 436, no. 1-2, pp. 806-814, 2012.

[24] Y. B. Feng, X. J. Zhao, F. Lv et al., "Optimization on preparation conditions of salidroside liposome and its immunological activity on PCV-2 in mice," Evidence-Based Complementary and Alternative Medicine, vol. 2015, p. 12, Article ID 178128, 2015.

[25] J. Xiao, J. Yao, L. Jia, C. Lin, and D. N. Zacks, “Protective effect of met12, a small peptide inhibitor of fas, on the retinal pigment epithelium and photoreceptor after sodium iodate injury," Investigative Opthalmology \& Visual Science, vol. 58, no. 3, pp. 1801-1810, 2017.

[26] X. Y. Lin, T. K. Ng, M. E. Brelén et al., "Continuous exposure to non-lethal doses of sodium iodate induces retinal pigment epithelial cell dysfunction," Scientific Reports, vol. 6, no. 1, Article ID 37279, 2016.

[27] K. Katsuji, Y. Katsuhiko, S. Nobuaki et al., "Morphologic characteristics of retinal degeneration induced by sodium iodate in mice," Current Eye Research, vol. 25, no. 6, pp. 373-379, 2002.

[28] Y. Li, P. Atmaca-Sonmez, C. L. Schanie, S. T. Ildstad, H. J. Kaplan, and V. Enzmann, "Endogenous bone marrowderived cells express retinal pigment epithelium cell markers and migrate to focal areas of RPE damage," Investigative Opthalmology \& Visual Science, vol. 48, no. 9, pp. 4321-4327, 2007.

[29] Z. Ildstad, J. Dai, J. He, C. Li, Y. Li, and Z. Q. Yin, "The influence of $\mathrm{NaIO}_{3}$-induced retinal degeneration on intraretinal layer and the changes of expression profile/morphology of DA-ACs and mRGCS," Molecular Neurobiology, vol. 47, no. 1, pp. 241-260, 2013.

[30] H. Li, K. Sugihara, C. Yamada, S. Tsutsumi, and Y. Iwaki, "Effect of estrogen on electroretinographic responses in streptozotocin-induced diabetic female rats," Experimental Eye Research, vol. 90, no. 5, pp. 591-597, 2010.

[31] S. Sandalon and R. Ofri, "The effect of topical anesthesia on the rat electroretinogram," Documenta Ophthalmologica, vol. 118, no. 2, pp. 101-108, 2009.

[32] Z. Wang, H. J. Mu, X. M. Zhang et al., "Lower irritation microemulsion-based rotigotine gel: formulation optimization and in vitro and in vivo studies," International Journal of Nanomedicine, vol. 10, pp. 633-644, 2015.

[33] S. Sun, R. Kislay, K. K. Rupinder, and K. R. Kanwar, "Evaluation of nanoformulated therapeutics in an ex-vivo bovine corneal irritation model," Toxicology in Vitro, vol. 29, no. 5, pp. 917-925, 2015.

[34] Code.org, Guideline for Local Irritation and Hemolysis of Traditional Chinese Medicine and Natural Medicine, Code.org, Seattle, WA, USA, 2019, http://www.cde.org.cn/ zdyz.do?method=largePage\&id=2088.

[35] J. E. Chastain, M. E. Sanders, M. A. Curtis et al., "Distribution of topical ocular nepafenac and its active metabolite amfenac to the posterior segment of the eye," Experimental Eye Research, vol. 145, pp. 58-67, 2016.

[36] C. Chemuturi, J.-L. Bourges, F. Valamanesh et al., "Novel micelle carriers for cyclosporin a topical ocular delivery: in vivo cornea penetration, ocular distribution and efficacy studies," European Journal of Pharmaceutics and Biopharmaceutics, vol. 81, no. 2, pp. 257-264, 2012.

[37] T. Trubitsyn, A. Isowaki, and H. Sakaki, "Ocular distribution of difluprednate ophthalmic emulsion $0.05 \%$ in rabbits," Journal of Ocular Pharmacology and Therapeutics, vol. 27, no. 1, pp. 43-49, 2011. 
[38] J. Shen, Y. Deng, X. Jin et al., “Thiolated nanostructured lipid carriers as a potential ocular drug delivery system for cyclosporine A: improving in vivo ocular distribution," International Journal of Pharmaceutics, vol. 402, no. 1-2, pp. 248-253, 2010.

[39] M. B. Du, S. Z. Liu, L. N. Liang, S. Shen, and K. Xu, "Comparison of pharmaceutical characterization and tissue distribution between chuanqi ophthalmic microemulsion in situ gel and normal in situ gel," Chinese Journal of Experimental Traditional Medical Formulae, vol. 25, no. 5, pp. 151-155, 2019.

[40] W. L. Yang and L. Zhang, "Normal range of temperature of cornea on ocular surface of healthy people in pudong new district of Shanghai," Journal of Shanghai Jiaotong University, vol. 30, no. 7, pp. 832-834, 2010.

[41] A. Vincent, A. G. Robson, and G. E. Holder, "Pathognomonic (diagnostic) ERGs a review and update," Retina, vol. 33, no. 1, pp. 5-12, 2013.

[42] A. Basile, "Operative versus nonoperative treatment of displaced intra-articular calcaneal fractures in elderly patients," The Journal of Foot and Ankle Surgery, vol. 49, no. 1, pp. 25-32, 2010.

[43] S. Yasuda, S. Kachi, S. Ueno, C.-H. Piao, and H. Terasaki, "Flicker electroretinograms before and after intravitreal ranibizumab injection in eyes with central retinal vein occlusion," Acta Ophthalmologica, vol. 93, no. 6, pp. e465-e468, 2015.

[44] R. N. Piao and P. M. Martin, "Data on the role of miR-144 in regulating fetal hemoglobin production in retinal pigmented epithelial cells," Data in Brief, vol. 28, Article ID 104874, 2020. 